\title{
The boundedness of commutators of rough $p$-adic fractional Hardy type operators on Herz-type spaces
}

\author{
Amjad Hussain ${ }^{1 *}$, Naqash Sarfraz ${ }^{2}$, Ilyas Khan ${ }^{3 *}$, Abdelaziz Alsubie ${ }^{4}$ and Nawaf N. Hamadneh ${ }^{4}$
}

\section{"Correspondence:}

ahabbasi123@yahoo.com; i.said@mu.edu.sa

'Department of Mathematics, Quaid-I-Azam University, 45320, Islamabad, 44000, Pakistan

${ }^{3}$ Department of Mathematics, College of Science Al-Zulfi,

Majmaah University, Al-Majmaah 11952, Saudi Arabia

Full list of author information is available at the end of the article

\begin{abstract}
In this paper, we obtain some inequalities about commutators of a rough $p$-adic fractional Hardy-type operator on Herz-type spaces when the symbol functions belong to two different function spaces.
\end{abstract}

MSC: 42B35; 26D15; 46B25; 47G10

Keywords: $p$-adic rough fractional Hardy operator; Commutators; $p$-adic Herz-type space; $p$-adic central BMO space; Lipschitz space

\section{Introduction}

During the last several decades, the $p$-adic analysis has cemented its role in the field of mathematical physics (see, for example, $[1,22,32,33])$. That stimulates researchers to pay attention to harmonic analysis on $p$-adic fields [18-21, 24, 30,31,35], which has direct implications in the stochastic process [2, 3], theoretical biology [6], and $p$-adic pseudodifferential equations [23, 34]. In continuation of the ongoing research, the present paper considers an extension of the investigation of $p$-adic Hardy-type operators discussed in [19-21, 25, 36, 37].

For every non-zero rational number $x$ there is a unique $\gamma=\gamma(x) \in \mathbb{Z}$ such that $x=p^{\gamma} m / n$, where $p \geq 2$ is a fixed prime number which is coprime to $m, n \in \mathbb{Z}$. We define a mapping $|\cdot|_{p}: \mathbb{Q} \rightarrow \mathbb{R}_{+}$as follows:

$$
|x|_{p}= \begin{cases}p^{-\gamma} & \text { if } x \neq 0 \\ 0 & \text { if } x=0 .\end{cases}
$$

The $p$-adic absolute value $|\cdot|_{p}$ has many properties of the usual real norm $|\cdot|$ with an additional non-Archimedean property,

$$
|x+y|_{p} \leq \max \left\{|x|_{p},|y|_{p}\right\} .
$$

The field of $p$-adic numbers, denoted by $\mathbb{Q}_{p}$, is the completion of rational numbers with respect to the $p$-adic absolute value $|\cdot|_{p}$. A $p$-adic number $x \in \mathbb{Q}_{p}$ can be written in the

(c) The Author(s) 2021. This article is licensed under a Creative Commons Attribution 4.0 International License, which permits use, sharing, adaptation, distribution and reproduction in any medium or format, as long as you give appropriate credit to the original author(s) and the source, provide a link to the Creative Commons licence, and indicate if changes were made. The images or other third party material in this article are included in the article's Creative Commons licence, unless indicated otherwise in a credit line to the material. If material is not included in the article's Creative Commons licence and your intended use is not permitted by statutory regulation or exceeds the permitted use, you will need to obtain permission directly from the copyright holder. To view a copy of this licence, visit http://creativecommons.org/licenses/by/4.0/. 
formal power series as [34]:

$$
x=p^{\gamma}\left(\beta_{0}+\beta_{1} p+\beta_{2} p^{2}+\cdots\right),
$$

where $\gamma \in \mathbb{Z}$ and $\beta_{i} \in\{0,1, \ldots, p-1\}, i=0,1,2, \ldots$ The $p$-adic absolute value ensures the convergence of series (1.2) in $\mathbb{Q}_{p}$, because the inequality $\left|p^{\gamma} \beta_{i} p^{i}\right|_{p} \leq p^{-\gamma-i}$ holds for all $\gamma \in \mathbb{Z}$ and $i \in \mathbb{N}$.

The $n$-dimensional vector space $\mathbb{Q}_{p}^{n}, n \geq 1$, consists of the vectors $\mathbf{x}=\left(x_{1}, x_{2}, \ldots, x_{n}\right)$, where $x_{j} \in \mathbb{Q}_{p}$ and $j=1,2, \ldots, n$, with the following absolute value:

$$
|\mathbf{x}|_{p}=\max _{1 \leq k \leq n}\left|x_{k}\right|_{p}
$$

For $\gamma \in \mathbb{Z}$ and $\mathbf{a}=\left(a_{1}, a_{2}, \ldots, a_{n}\right) \in \mathbb{Q}_{p}^{n}$, we denote by

$$
B_{\gamma}(\mathbf{a})=\left\{\mathbf{x} \in \mathbb{Q}_{p}^{n}:|\mathbf{x}-\mathbf{a}|_{p} \leq p^{\gamma}\right\}
$$

the closed ball with the center a and radius $p^{\gamma}$ and by

$$
S_{\gamma}(\mathbf{a})=\left\{\mathbf{x} \in \mathbb{Q}_{p}^{n}:|\mathbf{x}-\mathbf{a}|_{p}=p^{\gamma}\right\}
$$

the corresponding sphere. For $\mathbf{a}=\mathbf{0}$, we write $B_{\gamma}(\mathbf{0})=B_{\gamma}$, and $S_{\gamma}(\mathbf{0})=S_{\gamma}$. It is easy to see that the equalities

$$
\mathbf{a}_{0}+B_{\gamma}=B_{\gamma}\left(\mathbf{a}_{0}\right) \quad \text { and } \quad \mathbf{a}_{0}+S_{\gamma}=S_{\gamma}\left(\mathbf{a}_{0}\right)=B_{\gamma}\left(\mathbf{a}_{0}\right) \backslash B_{\gamma-1}\left(\mathbf{a}_{0}\right)
$$

hold for all $\mathbf{a}_{0} \in \mathbb{Q}_{p}^{n}$ and $\gamma \in \mathbb{Z}$.

Since $\mathbb{Q}_{p}^{n}$ is a locally compact commutative group under addition, there exists a unique Haar measure $d \mathbf{x}$ on $\mathbb{Q}_{p}^{n}$, such that

$$
\int_{B_{0}} d \mathbf{x}=\left|B_{0}\right|_{h}=1
$$

where $|B|_{h}$ denotes the Haar measure of measurable subset $B$ of $\mathbb{Q}_{p}^{n}$. Furthermore, a simple calculation shows that

$$
\left|B_{\gamma}(\mathbf{a})\right|_{h}=p^{n \gamma} \quad \text { and } \quad\left|S_{\gamma}(\mathbf{a})\right|_{h}=p^{n \gamma}\left(1-p^{-n}\right)
$$

hold for all $\mathbf{a} \in \mathbb{Q}_{p}^{n}$ and $\gamma \in \mathbb{Z}$.

The one-dimensional Hardy operator

$$
\mathcal{H} f(x)=\frac{1}{x} \int_{0}^{x} f(y) d y, \quad x>0,
$$

where $f: \mathbb{R}^{+} \rightarrow \mathbb{R}^{+}$is a measurable functions, was introduced by Hardy in [13]. This operator satisfies the inequality:

$$
\|\mathcal{H} f\|_{L^{q}\left(\mathbb{R}^{+}\right)} \leq \frac{q}{q-1}\|f\|_{L^{q}\left(\mathbb{R}^{+}\right)}, \quad 1<q<\infty
$$


where the constant $q /(q-1)$ is sharp. In [7], Faris proposed an extension of the operator $\mathcal{H}$ on higher dimensional Euclidean space $\mathbb{R}^{n}$ which is given by

$$
H f(\mathbf{x})=\frac{1}{|\mathbf{x}|^{n}} \int_{|\mathbf{y}| \leq|\mathbf{x}|} f(\mathbf{y}) d \mathbf{y}
$$

for $\mathbf{x}=\left(x_{1}, \ldots, x_{n}\right)$. In addition, Christ and Grafakos [4] obtained the exact value of the norm of operator $H$ defined by (1.5). For boundedness results for these operators on function spaces we refer to some recent publications including $[8,10,16,17,28,29,38]$.

On the other hand, the $n$-dimensional fractional $p$-adic Hardy operator

$$
H_{\alpha}^{p} f(\mathbf{x})=\frac{1}{|\mathbf{x}|_{p}^{n-\alpha}} \int_{|\mathbf{y}|_{p} \leq|\mathbf{x}|_{p}} f(\mathbf{y}) d \mathbf{y}
$$

was defined and studied for $f \in L_{1}^{\text {loc }}\left(\mathbb{Q}_{p}^{n}\right)$ and $0 \leq \alpha<n$ in [36]. When $\alpha=0$, the operator $H_{\alpha}^{p}$ transfers to the $p$-adic Hardy-type operator (see [10] for more details). Fu et al. in [9], fixed the optimal bounds of $p$-adic Hardy operator on $L^{q}\left(\mathbb{Q}_{p}^{n}\right)$. On the central Morrey space the $p$-adic Hardy-type operators and their commutators were discussed in [37]. In this connection see also [19, 21, 25].

There is still zero attention towards the rough Hardy operators on the $p$-adic linear spaces. Motivated by papers cited above and results of Fu et al. in [8], we define the special kind of $p$-adic rough fractional Hardy operator $H_{\Omega, \alpha}^{p}$ and its commutators as follows.

Definition 1.1 Let $f: \mathbb{Q}_{p}^{n} \rightarrow \mathbb{R}, b: \mathbb{Q}_{p}^{n} \rightarrow \mathbb{R}$ be measurable mappings and let $0<\alpha<n$. Then, for $\mathbf{x} \in \mathbb{Q}_{p}^{n} \backslash\{\mathbf{0}\}$, we define the rough $p$-adic fractional Hardy operator $H_{\Omega, \alpha}^{p}$ by

$$
H_{\Omega, \alpha}^{p} f(\mathbf{x})=\frac{1}{|\mathbf{x}|_{p}^{n-\alpha}} \int_{|\mathbf{y}|_{p} \leq|\mathbf{x}|_{p}} \Omega\left(|\mathbf{y}|_{p} \mathbf{y}\right) f(\mathbf{y}) d \mathbf{y}
$$

and its commutator $H_{\Omega, \alpha}^{p, b}$ by

$$
H_{\Omega, \alpha}^{p, b} f(\mathbf{x})=\frac{1}{|\mathbf{x}|_{p}^{n-\alpha}} \int_{|\mathbf{y}|_{p} \leq|\mathbf{x}|_{p}}(b(\mathbf{x})-b(\mathbf{y})) \Omega\left(|\mathbf{y}|_{p} \mathbf{y}\right) f(\mathbf{y}) d \mathbf{y}
$$

whenever

$$
\int_{|\mathbf{y}|_{p} \leq|\mathbf{x}|_{p}}\left|\Omega\left(|\mathbf{y}|_{p} \mathbf{y}\right) f(\mathbf{y})\right| d \mathbf{y}<\infty
$$

and

$$
\int_{|\mathbf{y}|_{p} \leq|\mathbf{x}|_{p}}\left|b(\mathbf{y}) \Omega\left(|\mathbf{y}|_{p} \mathbf{y}\right) f(\mathbf{y})\right| d \mathbf{y}<\infty,
$$

where $\Omega \in L^{s}\left(S_{0}(\mathbf{0})\right), 1 \leq s<\infty$.

Remark 1.2 Obviously

$$
\left\{|\mathbf{y}|_{p}: \mathbf{y} \in \mathbb{Q}_{p}^{n}\right\}=\left\{p^{\gamma}: \gamma \in \mathbb{Z}\right\} \cup\{0\}
$$


holds for every integer $n \geq 1$ and prime $p \geq 2$. Since the inclusion

$$
\{0\} \cup\left\{p^{\gamma}: \gamma \in \mathbb{Z}\right\} \subseteq \mathbb{Q}_{p}
$$

holds and $\mathbb{Q}_{p}^{n}$ is a linear space over field $\mathbb{Q}_{p}$, the product $|\mathbf{y}|_{p} \mathbf{y}$ is well defined. Moreover, if a non-zero $\mathbf{y} \in \mathbb{Q}_{p}^{n}$ has the form $\mathbf{y}=\left(y_{1}, \ldots, y_{n}\right)$ and

$$
y_{i}=p^{\gamma_{i}}\left(\beta_{0, i}+\beta_{1, i} p+\beta_{2, i} p^{2}+\cdots\right), \quad i=1, \ldots, n
$$

(see (1.2)), then there is $i_{0} \in\{1, \ldots, n\}$ such that

$$
\left|y_{i_{0}}\right|_{p}=p^{-\gamma_{i_{0}}} \geq p^{-\gamma_{i}}=\left|y_{i}\right|_{p}
$$

whenever $y_{i} \neq 0$. Using (1.3) we obtain $|\mathbf{y}|_{p}=p^{-\gamma_{i_{0}}}$. Now from (1.10) and (1.11) it follows that

$$
\left.\left.|| \mathbf{y}\right|_{p} \mathbf{y}\right|_{p}=\max _{\substack{1 \leq i \leq n \\ y_{i} \neq 0}}\left|p^{\gamma_{i}-\gamma_{i_{0}}}\right|_{p}=\max _{\substack{1 \leq i \leq n \\ y_{i} \neq 0}} p^{\gamma_{i_{0}}-\gamma_{i}}=p^{\gamma_{i_{0}}-\gamma_{i_{0}}}=1
$$

Thus, for every non-zero $\mathbf{y} \in \mathbb{Q}_{p}^{n}$, the vector $|\mathbf{y}|_{p} \mathbf{y}$ belongs to the sphere

$$
S_{0}(\mathbf{0})=\left\{\mathbf{y} \in \mathbb{Q}_{p}^{n}:|\mathbf{y}|_{p}=1\right\} \text {. }
$$

From (1.8) it directly follows that $H_{\Omega, \alpha}^{p} \in \mathbb{R}$ for every non-zero $\mathbf{x} \in \mathbb{Q}_{p}^{n}$ and using (1.8), (1.9) we have

$$
\begin{aligned}
\left|H_{\Omega, \alpha}^{p, b} f(\mathbf{x})\right| \leq & \frac{|b(\mathbf{x})|}{|\mathbf{x}|_{p}^{n-\alpha}} \int_{|\mathbf{y}|_{p} \leq|\mathbf{x}|_{p}}\left|\Omega\left(|\mathbf{y}|_{p} \mathbf{y}\right) f(\mathbf{y})\right| d \mathbf{y} \\
& +\frac{1}{|\mathbf{x}|_{p}^{n-\alpha}} \int_{|\mathbf{y}|_{p} \leq|\mathbf{x}|_{p}}\left|b(\mathbf{y}) \Omega\left(|\mathbf{y}|_{p} \mathbf{y}\right) f(\mathbf{y})\right| d \mathbf{y}<\infty
\end{aligned}
$$

for every $\mathbf{x} \in \mathbb{Q}_{p}^{n} \backslash\{\mathbf{0}\}$. Consequently, the operators $H_{\Omega, \alpha}^{p}$ and $H_{\Omega, \alpha}^{p, b}$ are well defined.

The aim of the current paper is to study the boundedness of $H_{\Omega, \alpha}^{p, b}$ on $p$-adic Herz-type spaces by considering the symbol function $b$ belonging to the $p$-adic $C M O$ and Lipschitz spaces. In Euclidean space $\mathbb{R}^{n}$, Herz spaces and Morrey-Herz spaces were firstly introduced in [14] and [26], respectively. For more recent developments in the said spaces we mention the articles $[15,27,39]$ and the references therein. Also, some operators with rough kernels defined on Euclidian space were recently studied on function spaces; see for example $[11,12]$. Before turning to our main results, let us recall the definitions of $p$-adic function spaces first.

Definition 1.3 ([9]) Suppose $1<q<\infty$. The $p$-adic central bounded mean oscillation $(\mathrm{CBMO})$ space $C \dot{M} O^{q}\left(\mathbb{Q}_{p}^{n}\right)$ is the set of all measurable functions $f: \mathbb{Q}_{p}^{n} \rightarrow \mathbb{R}$ which satisfy

$$
\|f\|_{C M O^{q}\left(\mathbb{Q}_{p}^{n}\right)}=\sup _{\gamma \in \mathbb{Z}}\left(\frac{1}{\left|B_{\gamma}\right|_{h}} \int_{B_{\gamma}}\left|f(\mathbf{x})-f_{B_{\gamma}}\right|^{q} d \mathbf{x}\right)^{1 / q}<\infty,
$$

where $f_{B_{\gamma}}=\frac{1}{\left|B_{\gamma}\right|_{h}} \int_{B_{\gamma}} f(\mathbf{x}) d \mathbf{x},\left|B_{\gamma}\right|_{h}$ is the Haar measure of $B_{\gamma}$. 
Definition 1.4 ([9]) Suppose $0<r<\infty, 0<q<\infty$ and $\beta \in \mathbb{R}$. The homogeneous $p$-adic Herz space $\dot{K}_{q}^{\beta, r}\left(\mathbb{Q}_{p}^{n}\right)$ is defined by

$$
\dot{K}_{q}^{\beta, r}\left(\mathbb{Q}_{p}^{n}\right)=\left\{f \in L^{q}\left(\mathbb{Q}_{p}^{n}\right):\|f\|_{\dot{K}_{q}^{\beta, r}\left(\mathbb{Q}_{p}^{n}\right)}<\infty\right\},
$$

where

$$
\|f\|_{\dot{K}_{q}^{\beta, r}\left(\mathbb{Q}_{p}^{n}\right)}=\left(\sum_{k=-\infty}^{\infty} p^{k \beta r}\left\|f \chi_{k}\right\|_{L^{q}\left(\mathbb{Q}_{p}^{n}\right)}^{r}\right)^{1 / r}
$$

and $\chi_{k}$ is the characteristic function of $S_{k}$.

Obviously, the equalities $\dot{K}_{q}^{0, q}\left(\mathbb{Q}_{p}^{n}\right)=L^{q}\left(\mathbb{Q}_{p}^{n}\right)$ and $\dot{K}_{q}^{\beta / q, q}\left(\mathbb{Q}_{p}^{n}\right)=L^{q}\left(|\mathbf{x}|_{p}^{\beta}\right)$ hold.

Definition 1.5 ([5]) Suppose $0<r<\infty, 0<q<\infty, \beta \in \mathbb{R}$ and $\lambda \geq 0$. The homogeneous $p$-adic Morrey-Herz space is defined by

$$
M \dot{K}_{q, r}^{\beta, \lambda}\left(\mathbb{Q}_{p}^{n}\right)=\left\{f \in L_{\mathrm{loc}}^{q}\left(\mathbb{Q}_{p}^{n} \backslash\{0\}\right):\|f\|_{M \dot{K}_{r, q}^{\beta, \lambda}\left(\mathbb{Q}_{p}^{n}\right)}<\infty\right\},
$$

where

$$
\|f\|_{M \dot{K}_{r, q}^{\beta, \lambda}\left(\mathbb{Q}_{p}^{n}\right)}=\sup _{k_{0} \in \mathbb{Z}} p^{-k_{0} \lambda}\left(\sum_{k=-\infty}^{k_{0}} p^{k \beta r}\left\|f \chi_{k}\right\|_{L^{q}\left(\mathbb{Q}_{p}^{n}\right)}^{r}\right)^{1 / r} .
$$

It is evident that $M \dot{K}_{r, q}^{\beta, 0}\left(\mathbb{Q}_{p}^{n}\right)=\dot{K}_{q}^{\beta, r}\left(\mathbb{Q}_{p}^{n}\right)$ and $M \dot{K}_{q, q}^{\beta / q, 0}\left(\mathbb{Q}_{p}^{n}\right)=L^{q}\left(|\mathbf{x}|_{p}^{\alpha}\right)$.

Definition $1.6([5])$ Suppose $\delta$ is a positive real number. The Lipschitz space $\Lambda_{\delta}\left(\mathbb{Q}_{p}^{n}\right)$ is defined to be the space of all measurable function $f$ on $\mathbb{Q}_{p}^{n}$ such that

$$
\|f\|_{\Lambda_{\delta}\left(\mathbb{Q}_{p}^{n}\right)}=\sup _{\mathbf{x}, \mathbf{h} \in \mathbb{Q}_{p}^{n}, \mathbf{h} \neq 0} \frac{|f(\mathbf{x}+\mathbf{h})-f(\mathbf{x})|}{|\mathbf{h}|_{p}^{\delta}}<\infty .
$$

\section{$2 C B M O$ estimates for commutators of $p$-adic rough fractional Hardy operator}

The present section discusses the boundedness of $p$-adic rough fractional Hardy operator on $p$-adic Herz-type spaces. We begin this section with the following useful lemma.

Lemma 2.1 ([36]) Suppose b is a $C M O^{1}\left(\mathbb{Q}_{p}^{n}\right)$ function and suppose $i, j \in \mathbb{Z}$. Then the inequality

$$
\left|b(\mathbf{y})-b_{B_{j}}\right| \leq\left|b(\mathbf{y})-b_{B_{i}}\right|+p^{n}|i-j|\|b\|_{C M O^{1}\left(\mathbb{Q}_{p}^{n}\right)}
$$

holds.

Remark 2.2 From now on the letter $C$ indicates a positive constant which may vary from line to line. 
Theorem 2.3 Let $0<r_{1} \leq r_{2}<\infty, 1 \leq q_{1}, q_{2}<\infty$. Also, let $\frac{1}{q_{1}}-\frac{1}{q_{2}}=\frac{\alpha}{n}, q_{1}^{\prime}<s<\infty, \frac{1}{q_{1}^{\prime}}-\frac{1}{t}=$ $\frac{1}{s}$. If $\beta<\frac{n}{t}$, then the inequality

$$
\left\|H_{\Omega, \alpha}^{p, b} f\right\|_{\dot{K}_{q 2}^{\beta, r_{2}}\left(\mathbb{Q}_{p}^{n}\right)} \leq C\|f\|_{\dot{K}_{q 1}^{\beta, r}{ }_{\left(\mathbb{Q}_{p}^{n}\right.}^{n}}
$$

holds for all $\Omega \in L^{s}\left(S_{\mathbf{0}}(\mathbf{0})\right), b \in C M O^{\max \left\{q_{2}, t\right\}}\left(\mathbb{Q}_{p}^{n}\right)$, and $f \in L_{\mathrm{loc}}^{q_{1}}\left(\mathbb{Q}_{p}^{n}\right)$.

Proof of Theorem 2.3 For the sake of brevity, we write

$$
\sum_{j=-\infty}^{\infty} f(\mathbf{x}) \chi_{j}(\mathbf{x})=\sum_{j=-\infty}^{\infty} f_{j}(\mathbf{x})
$$

Since

$$
\begin{aligned}
\left\|\left(H_{\Omega, \alpha}^{p, b} f\right) \chi_{k}\right\|_{L^{q_{2}\left(\mathbb{Q}_{p}^{n}\right)}}^{q_{2}}= & \int_{S_{k}}|\mathbf{x}|_{p}^{-q_{2}(n-\alpha)}\left|\int_{|\mathbf{y}|_{p} \leq|\mathbf{x}|_{p}} \Omega\left(|\mathbf{y}|_{p} \mathbf{y}\right) f(\mathbf{y})(b(\mathbf{x})-b(\mathbf{y})) d \mathbf{y}\right|^{q_{2}} d \mathbf{x} \\
\leq & C p^{-k q_{2}(n-\alpha)} \int_{S_{k}}\left(\int_{|\mathbf{y}|_{p} \leq p^{k}}\left|\Omega\left(|\mathbf{y}|_{p} \mathbf{y}\right) f(\mathbf{y})(b(\mathbf{x})-b(\mathbf{y}))\right| d \mathbf{y}\right)^{q_{2}} d \mathbf{x} \\
= & C p^{-k q_{2}(n-\alpha)} \int_{S_{k}}\left(\sum_{j=-\infty}^{k} \int_{S_{j}}\left|f(\mathbf{y}) \Omega\left(p^{j} \mathbf{y}\right)(b(\mathbf{x})-b(\mathbf{y}))\right| d \mathbf{y}\right)^{q_{2}} d \mathbf{x} \\
\leq & C p^{-k q_{2}(n-\alpha)} \int_{S_{k}}\left(\sum_{j=-\infty}^{k} \int_{S_{j}}\left|f(\mathbf{y}) \Omega\left(p^{j} \mathbf{y}\right)\left(b(\mathbf{x})-b_{B_{k}}\right)\right| d \mathbf{y}\right)^{q_{2}} d \mathbf{x} \\
& +C p^{-k q_{2}(n-\alpha)} \int_{S_{k}}\left(\sum_{j=-\infty}^{k} \int_{S_{j}}\left|f(\mathbf{y}) \Omega\left(p^{j} \mathbf{y}\right)\left(b(\mathbf{y})-b_{B_{k}}\right)\right| d \mathbf{y}\right)^{q_{2}} d \mathbf{x} \\
= & I+I I .
\end{aligned}
$$

For $j, k \in \mathbb{Z}$ with $j \leq k$, we get

$$
\int_{S_{j}}\left|\Omega\left(p^{j} \mathbf{y}\right)\right|^{s} d \mathbf{y}=\int_{|\mathbf{z}|_{p}=1}|\Omega(\mathbf{z})|^{s} p^{j n} d \mathbf{z} \leq C p^{k n}
$$

Note that $\frac{1}{q_{1}}+\frac{1}{q_{2}}=\frac{\alpha}{n}$ and $\frac{1}{q_{1}}+\frac{1}{s}+\frac{1}{t}=1$, where $\frac{1}{t}=\frac{1}{q_{1}^{\prime}}-\frac{1}{s}$. Applying Hölder's inequality we have

$$
\begin{aligned}
& I \leq C p^{-k q_{2}(n-\alpha)} \int_{B_{k}}\left|b(\mathbf{x})-b_{B_{k}}\right|^{q_{2}} \\
& \times\left\{\sum_{j=-\infty}^{k}\left(\int_{S_{j}}|f(\mathbf{y})|^{q_{1}} d \mathbf{y}\right)^{1 / q_{1}}\left(\int_{S_{j}}\left|\Omega\left(p^{j} \mathbf{y}\right)\right|^{s} d \mathbf{y}\right)^{1 / s} p^{j n\left(1 / q_{1}^{\prime}-1 / s\right)}\right\}^{q_{2}} d \mathbf{x} \\
& \leq C\|b\|_{C M O^{q_{2}}\left(\mathbb{Q}_{p}^{n}\right)}^{q_{2}} p^{k n-k q_{2}(n-\alpha)}\left\{\sum_{j=-\infty}^{k} p^{j n\left(1 / q_{1}^{\prime}-1 / s\right)} p^{k n / s}\left\|f_{j}\right\|_{L^{q_{1}}\left(\mathbb{Q}_{p}^{n}\right)}\right\}^{q_{2}} \\
& =C\|b\|_{C M O^{q_{2}}\left(\mathbb{Q}_{p}^{n}\right)}^{q_{2}}\left\{\sum_{j=-\infty}^{k} p^{(j-k) n\left(1 / q_{1}^{\prime}-1 / s\right)}\left\|f_{j}\right\|_{L^{q_{1}}\left(\mathbb{Q}_{p}^{n}\right)}\right\}^{q_{2}} .
\end{aligned}
$$


Hussain et al. Journal of Inequalities and Applications

(2021) $2021: 123$

Page 7 of 13

Lemma 2.1 will be helpful for estimating $I I$. Thus

$$
\begin{aligned}
I I \leq & C p^{-k q_{2}(n-\alpha)} \int_{S_{k}}\left(\sum_{j=-\infty}^{k} \int_{S_{j}}\left|f(\mathbf{y}) \Omega\left(p^{j} \mathbf{y}\right)\left(b(\mathbf{y})-b_{B_{j}}\right)\right| d \mathbf{y}\right)^{q_{2}} d \mathbf{x} \\
& +C\|b\|_{C M O^{1}\left(\mathbb{Q}_{p}^{n}\right)}^{q_{2}} p^{-k q_{2}(n-\alpha)} \int_{S_{k}}\left(\sum_{j=-\infty}^{k}(k-j) \int_{S_{j}}\left|f(\mathbf{y}) \Omega\left(p^{j} \mathbf{y}\right)\right| d \mathbf{y}\right)^{q_{2}} d \mathbf{x} \\
= & I_{1}+I I_{2} .
\end{aligned}
$$

We use Hölder's inequality to estimate $I_{1}$. We have

$$
\begin{aligned}
I_{1} \leq & C p^{-k q_{2}(n-\alpha)} \int_{S_{k}}\left\{\sum_{j=-\infty}^{k}\left(\int_{S_{j}}\left|b(\mathbf{y})-b_{B_{j}}\right|^{t} d \mathbf{y}\right)^{1 / t}\right. \\
& \left.\times\left(\int_{S_{j}}\left|\Omega\left(p^{j} \mathbf{y}\right)\right|^{s} d \mathbf{y}\right)^{1 / s}\left(\int_{S_{j}}|f(\mathbf{y})|^{q_{1}} d \mathbf{y}\right)^{1 / q_{1}}\right\}^{q_{2}} d \mathbf{x} \\
\leq & \|b\|_{C M O^{t}\left(\mathbb{Q}_{p}^{n}\right)}^{q_{2}} \sum_{j=-\infty}^{k}\left\{p^{-k n / q_{1}^{\prime}} p^{k n / s} p^{j n / t}\left(\frac{1}{\left|B_{j}\right|_{H}} \int_{B_{j}}\left|b(\mathbf{y})-b_{B_{j}}\right|^{t}\right)^{1 / t}\left\|f_{j}\right\|_{L^{q_{1}\left(\mathbb{Q}_{p}^{n}\right)}}\right\}^{q_{2}} \\
= & C\|b\|_{C M O^{t}\left(\mathbb{Q}_{p}^{n}\right)}^{q_{2}}\left\{\sum_{j=-\infty}^{k} p^{(j-k) n\left(1 / q_{1}^{\prime}-1 / s\right)}\left\|f_{j}\right\|_{L^{q_{1}}\left(\mathbb{Q}_{p}^{n}\right)}\right\}^{q_{2}} .
\end{aligned}
$$

In a similar fashion we can estimate $\mathrm{II}_{2}$. Using Hölder's inequality we have

$$
\begin{aligned}
I I_{2} \leq & C\|b\|_{C M O^{1}\left(\mathbb{Q}_{p}^{n}\right)}^{q_{2}} p^{-k q_{2}(n-\alpha)} \\
& \times \int_{S_{k}}\left\{\sum_{j=-\infty}^{k}(k-j)\left(\int_{S_{j}}|f(\mathbf{y})|^{q_{1}} d \mathbf{y}\right)^{1 / q_{1}}\left(\int_{S_{j}}\left|\Omega\left(p^{j} \mathbf{y}\right)\right|^{s} d \mathbf{y}\right)^{1 / s} p^{j n / t}\right\}^{q_{2}} d \mathbf{x} \\
= & C\|b\|_{C M O^{1}\left(\mathbb{Q}_{p}^{n}\right)}^{q_{2}}\left(\sum_{j=-\infty}^{k}(k-j) p^{(j-k) n\left(1 / q_{1}^{\prime}-1 / s\right)}\left\|f_{j}\right\|_{L^{q_{1}}\left(\mathbb{Q}_{p}^{n}\right)}\right)^{q_{2}} .
\end{aligned}
$$

From (2.3), (2.5) and (2.6) together with the Jensen inequality, we have

$$
\begin{aligned}
\left\|H_{\Omega, \alpha}^{p, b} f\right\|_{\dot{K}_{q 2}^{\beta, r_{2}}\left(\mathbb{Q}_{p}^{n}\right)} & \left(\sum_{k=-\infty}^{\infty} p^{k \beta r_{2}}\left\|\left(H_{\Omega, \alpha}^{p, b} f\right) \chi_{k}\right\|_{L^{q_{2}\left(\mathbb{Q}_{p}^{n}\right)}}^{r_{2}}\right)^{1 / r_{2}} \\
\leq & \left(\sum_{k=-\infty}^{\infty} p^{k \beta r_{1}}\left\|\left(H_{\Omega, \alpha}^{p, b} f\right) \chi_{k}\right\|_{L^{q_{2}}\left(\mathbb{Q}_{p}^{n}\right)}^{r_{1}}\right)^{1 / r_{1}} \\
\leq & C\|b\|_{C M O^{q_{2}}\left(\mathbb{Q}_{p}^{n}\right)}\left(\sum_{k=-\infty}^{\infty} p^{k \beta r_{1}}\left(\sum_{j=-\infty}^{k} p^{(j-k) n / t}\left\|f_{j}\right\|_{L^{q_{1}}\left(\mathbb{Q}_{p}^{n}\right)}\right)^{r_{1}}\right)^{1 / r_{1}} \\
& +C\|b\|_{C M O^{t}\left(\mathbb{Q}_{p}^{n}\right)}\left(\sum_{k=-\infty}^{\infty} p^{k \beta r_{1}}\left(\sum_{j=-\infty}^{k} p^{(j-k) n / t}\left\|f_{j}\right\|_{L^{q_{1}}\left(\mathbb{Q}_{p}^{n}\right)}\right)^{r_{1}}\right)^{1 / r_{1}}
\end{aligned}
$$




$$
\begin{aligned}
& +C\|b\|_{C M O^{1}\left(\mathbb{Q}_{p}^{n}\right)}\left(\sum_{k=-\infty}^{\infty} p^{k \beta r_{1}}\left(\sum_{j=-\infty}^{k}(k-j) p^{(j-k) n / t}\left\|f_{j}\right\|_{L^{q_{1}}\left(\mathbb{Q}_{p}^{n}\right)}\right)^{r_{1}}\right)^{1 / r_{1}} \\
= & J .
\end{aligned}
$$

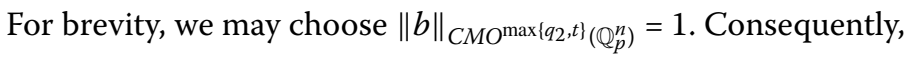

$$
J \leq C\left(\sum_{k=-\infty}^{\infty} p^{k \beta r_{1}}\left(\sum_{j=-\infty}^{k}(k-j) p^{(j-k) n / t}\left\|f_{j}\right\|_{L^{q_{1}}\left(\mathbb{Q}_{p}^{n}\right)}\right)^{r_{1}}\right)^{1 / r_{1}} .
$$

Case 1: When $0<r_{1} \leq 1$, we have

$$
\begin{aligned}
J^{r_{1}} & =C \sum_{k=-\infty}^{\infty} p^{k \beta r_{1}}\left(\sum_{j=-\infty}^{k}(k-j) p^{(j-k) n / t}\left\|f_{j}\right\|_{L^{q_{1}}\left(\mathbb{Q}_{p}^{n}\right)}\right)^{r_{1}} \\
& =C \sum_{k=-\infty}^{\infty}\left(\sum_{j=-\infty}^{k} p^{j \beta}\left\|f_{j}\right\|_{L^{q_{1}}\left(\mathbb{Q}_{p}^{n}\right)}(k-j) p^{(j-k)(n / t-\beta)}\right)^{r_{1}} \\
& \leq C \sum_{k=-\infty}^{\infty} \sum_{j=-\infty}^{k} p^{j \beta r_{1}}\left\|f_{j}\right\|_{L^{q_{1}}}^{r_{1}}\left(\mathbb{Q}_{p}^{n}\right) \\
& =C \sum_{k=-\infty}^{\infty} p^{j \beta r_{1}}\left\|f_{j}\right\|_{L^{q_{1}}}^{r_{1}}\left(\mathbb{Q}_{p}^{n}\right) \\
& \sum_{k=j}^{\infty}(k-j)^{r_{1}} p^{(j-k)(n / t-\beta) r_{1}} \\
& =C\|f\|_{\dot{K}_{q_{1}}^{\beta, r_{1}}}^{r_{1}} p_{\left.\mathbb{Q}_{p}^{n}\right)} \cdot
\end{aligned}
$$

Case 2: When $r_{1}>1$, applying Hölder's inequality we get

$$
\begin{aligned}
J^{r_{1}}= & C \sum_{k=-\infty}^{\infty}\left(\sum_{j=-\infty}^{k} p^{j \beta}\left\|f_{j}\right\|_{L^{q_{1}}\left(\mathbb{Q}_{p}^{n}\right)}(k-j) p^{(j-k)(n / t-\beta)}\right)^{r_{1}} \\
\leq & C \sum_{k=-\infty}^{\infty} \sum_{j=-\infty}^{k} p^{j \beta r_{1}}\left\|f_{j}\right\|_{L^{q_{1}}\left(\mathbb{Q}_{p}^{n}\right)}^{r_{1}} p^{(j-k)(n / t-\beta) r_{1} / 2} \\
& \times\left(\sum_{j=-\infty}^{k}(k-j)^{r_{1}^{\prime}} p^{(j-k)(n / t-\beta) r_{1}^{\prime} / 2}\right)^{r_{1} / r_{1}^{\prime}} \\
= & C \sum_{k=-\infty}^{\infty} p^{j \beta r_{1}}\left\|f_{j}\right\|_{L^{q_{1}}}^{r_{1}}\left(\mathbb{Q}_{p}^{n}\right) \\
= & C\|f\|_{k=j}^{r_{1} r_{q_{1}}^{\beta, r_{1}}} p_{\left.\mathbb{Q}_{p}^{n}\right)^{n}} \cdot
\end{aligned}
$$

The proof of Theorem 2.3 is thus completed.

Theorem 2.4 Let $0<r_{1} \leq r_{2}<\infty, 1 \leq q_{1}, q_{2}<\infty$. Also, let $\frac{1}{q_{1}}-\frac{1}{q_{2}}=\frac{\alpha}{n}, q_{1}^{\prime}<s<\infty, \frac{1}{q_{1}^{\prime}}-\frac{1}{t}=$ $\frac{1}{s}$, and $\lambda>0$. If $\beta<\frac{n}{t}+\lambda$, then the inequality

$$
\left\|H_{\Omega, \alpha}^{p, b} f\right\|_{M \dot{K}_{r_{2}, q_{2}}^{\beta, \lambda}\left(\mathbb{Q}_{p}^{n}\right)} \leq C\|f\|_{M \dot{K}_{r_{1}, q_{1}}^{\beta, \lambda}\left(\mathbb{Q}_{p}^{n}\right)}
$$

holds for all $\Omega \in L^{s}\left(S_{\mathbf{0}}(\mathbf{0})\right), b \in C M O^{\max \left\{q_{2}, t\right\}}\left(\mathbb{Q}_{p}^{n}\right)$ and $f \in L_{\mathrm{loc}}^{q_{1}}\left(\mathbb{Q}_{p}^{n}\right)$. 
Proof of Theorem 2.4 From the proof of Theorem 2.3 and

$$
\left\|\left(H_{\Omega, \alpha}^{p, b} f\right) \chi_{k}\right\|_{L^{q_{2}\left(\mathbb{Q}_{p}^{n}\right)}} \leq C \sum_{j=-\infty}^{k}(k-j) p^{\frac{(j-k) n}{t}}\left\|f_{j}\right\|_{L^{q_{1}}\left(\mathbb{Q}_{p}^{n}\right)}
$$

together with the definition of a Morrey-Herz space, the Jensen inequality, $\beta<n / t+\lambda$, $\lambda>0$ and $1<r_{1}<\infty$, it follows that

$$
\begin{aligned}
& \left\|H_{\Omega, \alpha}^{p, b} f\right\|_{M \dot{K}_{r_{2}, q_{2}}^{\beta, \lambda}\left(\mathbb{Q}_{p}^{n}\right)} \\
& =\sup _{k_{0} \in \mathbb{Z}} p^{-k_{0} \lambda}\left(\sum_{k=-\infty}^{k_{0}} p^{k \beta r_{2}}\left\|\left(H_{\Omega, \alpha}^{p, b} f\right) \chi_{k}\right\|_{L^{q 2}\left(\mathbb{Q}_{p}^{n}\right)}^{r_{2}}\right)^{1 / r_{2}} \\
& \leq \sup _{k_{0} \in \mathbb{Z}} p^{-k_{0} \lambda}\left(\sum_{k=-\infty}^{k_{0}} p^{k \beta r_{1}}\left\|\left(H_{\Omega, \alpha}^{p, b} f\right) \chi_{k}\right\|_{L^{q_{2}\left(\mathbb{Q}_{p}^{n}\right)}}^{r_{1}}\right)^{1 / r_{1}} \\
& \leq C \sup _{k_{0} \in \mathbb{Z}} p^{-k_{0} \lambda}\left(\sum_{k=-\infty}^{k_{0}} p^{k \beta r_{1}}\left(\sum_{j=-\infty}^{k}(k-j) p^{\frac{(j-k) n}{t}}\left\|f_{j}\right\|_{L^{q_{1}}\left(\mathbb{Q}_{p}^{n}\right)}\right)^{r_{1}}\right)^{1 / r_{1}} \\
& \leq C \sup _{k_{0} \in \mathbb{Z}} p^{-k_{0} \lambda}\left(\sum _ { k = - \infty } ^ { k _ { 0 } } \left(\sum_{j=-\infty}^{k} p^{k \beta}(k-j) p^{\frac{(j-k) n}{t}} p^{-j \beta} p^{j \lambda} p^{j \lambda}\right.\right. \\
& \left.\left.\times\left(\sum_{l=-\infty}^{j} p^{l \beta r_{1}}\left\|f_{j}\right\|_{L^{q_{1}\left(\mathbb{Q}_{p}^{n}\right)}}^{r_{1}}\right)^{1 / r_{1}}\right)^{r_{1}}\right)^{1 / r_{1}} \\
& \leq C \sup _{k_{0} \in \mathbb{Z}} p^{-k_{0} \lambda}\left(\sum_{k=-\infty}^{k_{0}} p^{k \lambda r_{1}}\left(\sum_{j=-\infty}^{k}(k-j) p^{(j-k)(n / t-\beta+\lambda)}\|f\|_{M \dot{K}_{r_{1}, q_{1}}^{\beta, \lambda}\left(\mathbb{Q}_{p}^{n}\right)}\right)^{r_{1}}\right)^{1 / r_{1}} \\
& \leq C\|f\|_{M \dot{K}_{r_{1}, q_{1}}^{\beta, \lambda}\left(\mathbb{Q}_{p}^{n}\right)} .
\end{aligned}
$$

\section{Lipschitz estimates for commutators of $p$-adic rough fractional Hardy} operator

The current section deals with the boundedness for the commutators of $p$-adic rough fractional Hardy operator on homogeneous $p$-adic Herz-type spaces by considering the symbol function from Lipschitz space. We open the discussion for this section from the following lemma.

Lemma 3.1 Suppose $f \in \Lambda_{\delta}\left(\mathbb{Q}_{p}^{n}\right)$ and $0<\delta<1$, then

$$
|f(\mathbf{x})-f(\mathbf{y})| \leq|\mathbf{x}-\mathbf{y}|_{p}^{\delta}\|f\|_{\Lambda_{\delta}\left(\mathbb{Q}_{p}^{n}\right)}
$$

Proof Proof immediately follows from Definition 1.6.

Theorem 3.2 Let $1 \leq q_{1}, q_{2}<\infty, 0<r_{1} \leq r_{2}<\infty$. Also, let $\frac{1}{q_{1}}-\frac{1}{q_{2}}=\frac{\delta+\alpha}{n}, q_{1}^{\prime}<s<\infty$, $\frac{1}{q_{1}^{\prime}}-\frac{1}{t}=\frac{1}{s}$, and $0<\delta<1$. If $\beta<n\left(\frac{1}{q_{1}^{\prime}}-\frac{1}{s}\right)$, then the inequality

$$
\left\|H_{\Omega, a}^{p, b} f\right\|_{\dot{K}_{q 2}^{\beta, r_{2}}\left(\mathbb{Q}_{p}^{n}\right)} \leq C\|f\|_{\dot{K}_{q 1}^{\beta, r_{1}}\left(\mathbb{Q}_{p}^{n}\right)}
$$

holds for all $\Omega \in L^{s}\left(S_{\mathbf{0}}(\mathbf{0})\right), b \in \Lambda_{\delta}\left(\mathbb{Q}_{p}^{n}\right)$, and $f \in L_{\mathrm{loc}}^{q_{1}}\left(\mathbb{Q}_{p}^{n}\right)$. 
Proof of Theorem 3.2 By Hölder's inequality along with Lemma 3.1, we have

$$
\begin{aligned}
& \left\|\left(H_{\Omega, \alpha}^{p, b} f\right) \chi_{k}\right\|_{L^{q_{2}}\left(\mathbb{Q}_{p}^{n}\right)}^{q_{2}} \\
& =\int_{S_{k}}|\mathbf{x}|_{p}^{-q_{2}(n-\alpha)}\left|\int_{|\mathbf{y}|_{p} \leq|\mathbf{x}|_{p}} \Omega\left(|\mathbf{y}|_{p} \mathbf{y}\right) f(\mathbf{y})(b(\mathbf{x})-b(\mathbf{y})) d \mathbf{y}\right|^{q_{2}} d \mathbf{x} \\
& \leq C p^{-k q_{2}(n-\alpha)} \int_{S_{k}}\left(\int_{|\mathbf{y}|_{p} \leq p^{k}}\left|\Omega\left(|\mathbf{y}|_{p} \mathbf{y}\right) f(\mathbf{y})(b(\mathbf{x})-b(\mathbf{y}))\right| d \mathbf{y}\right)^{q 2} d \mathbf{x} \\
& \leq C p^{-k q_{2}(n-\alpha)}\|b\|_{\Lambda_{\delta}\left(\mathbb{Q}_{p}^{n}\right)}^{q_{2}} \int_{S_{k}}\left(\sum_{j=-\infty}^{k} \int_{S_{j}}\left|\Omega\left(p^{j} \mathbf{y}\right) f(\mathbf{y})\right||\mathbf{x}-\mathbf{y}|_{p}^{\delta} d \mathbf{y}\right)^{q_{2}} d \mathbf{x} \\
& \leq C p^{-k q_{2}(n-\alpha-\delta)}\|b\|_{\Lambda_{\delta}\left(\mathbb{Q}_{p}^{n}\right)}^{q_{2}} \int_{S_{k}}\left(\sum_{j=-\infty}^{k} \int_{S_{j}}\left|\Omega\left(p^{j} \mathbf{y}\right) f(\mathbf{y})\right| d \mathbf{y}\right)^{q_{2}} d \mathbf{x} \\
& \leq C\|b\|_{\Lambda_{\delta}\left(\mathbb{Q}_{p}^{n}\right)}^{q_{2}} p^{-k q_{2}(n-\alpha-\delta)+k n}\left(\sum_{j=-\infty}^{k}\left(\int_{S_{j}}\left|\Omega\left(p^{j} \mathbf{y}\right)\right|^{s} d \mathbf{y}\right)^{1 / s}\right. \\
& \left.\times\left(\int_{S_{j}}|f(\mathbf{y})|^{q_{1}} d \mathbf{y}\right)^{1 / q_{1}}\left(\int_{S_{j}} d \mathbf{y}\right)^{1-1 / q-1 / s}\right)^{q_{2}} \\
& =I \text {. }
\end{aligned}
$$

By virtue of (2.2), inequality (3.1) takes the following form:

$$
\begin{aligned}
I & \leq C\|b\|_{\Lambda_{\delta}\left(\mathbb{Q}_{p}^{n}\right)}^{q_{2}} p^{-k q_{2}(n-\alpha-\delta)+k n}\left(\sum_{j=-\infty}^{k} p^{k n / s+j n\left(1 / q_{1}^{\prime}-1 / s\right)}\left\|f_{j}\right\|_{L^{q_{1}}\left(\mathbb{Q}_{p}^{n}\right)}\right)^{q_{2}} \\
& \leq C\|b\|_{\Lambda_{\delta}\left(\mathbb{Q}_{p}^{n}\right)}^{q_{2}}\left(\sum_{j=-\infty}^{k} p^{(j-k) n\left(1 / q^{\prime}-1 / s\right)}\left\|f_{j}\right\|_{L^{q_{1}\left(\mathbb{Q}_{p}^{n}\right)}}\right)^{q_{2}} .
\end{aligned}
$$

For the sake of brevity, we take $\|b\|_{\Lambda_{\delta}\left(\mathbb{Q}_{p}^{n}\right)}^{q_{2}}=1$. Now, by definition of Herz spaces and the Jensen inequality, it follows that

$$
\begin{aligned}
\left\|H_{\Omega, \alpha}^{p, b} f\right\|_{\dot{K}_{q_{2}}^{\beta, r_{2}\left(\mathbb{Q}_{p}^{n}\right)}}^{r_{1}} & =\left(\sum_{k=-\infty}^{\infty} p^{k \beta r_{2}}\left\|\left(H_{\Omega, \alpha}^{p, b} f\right) \chi_{k}\right\|_{L^{q_{2}\left(\mathbb{Q}_{p}^{n}\right)}}^{r_{2}}\right)^{r_{1} / r_{2}} \\
& \leq \sum_{k=-\infty}^{\infty} p^{k \beta r_{1}}\left\|\left(H_{\Omega, \alpha}^{p, b} f\right) \chi_{k}\right\|_{L^{q_{2}\left(\mathbb{Q}_{p}^{n}\right)}}^{r_{1}} \\
& \leq C \sum_{k=-\infty}^{\infty} p^{k \beta r_{1}}\left(\sum_{j=-\infty}^{k} p^{(j-k) n\left(1 / q_{1}^{\prime}-1 / s\right)}\left\|f_{j}\right\|_{L^{q_{1}\left(\mathbb{Q}_{p}^{n}\right)}}\right)^{r_{1}} \\
& =C \sum_{k=-\infty}^{\infty}\left(\sum_{j=-\infty}^{k} p^{j \beta} p^{(j-k)\left(n / q_{1}^{\prime}-n / s-\beta\right)}\left\|f_{j}\right\|_{L^{q_{1}\left(\mathbb{Q}_{p}^{n}\right)}}\right)^{r_{1}} .
\end{aligned}
$$


Case 1: If $0<r_{1} \leq 1$, then

$$
\begin{aligned}
\left\|H_{\Omega, \alpha}^{p, b} f\right\|_{\dot{K}_{q_{2}}^{\beta, r_{2}}\left(\mathbb{Q}_{p}^{n}\right)}^{r_{1}} & \leq C \sum_{k=-\infty}^{\infty} \sum_{j=-\infty}^{k} p^{j \beta r_{1}} p^{(j-k)\left(n / q_{1}^{\prime}-n / s-\beta\right) r_{1}}\left\|f_{j}\right\|_{L^{q_{1}}\left(\mathbb{Q}_{p}^{n}\right)}^{r_{1}} \\
& =C \sum_{j=-\infty}^{\infty} p^{j \beta r_{1}}\left\|f_{j}\right\|_{L^{q_{1}}\left(\mathbb{Q}_{p}^{n}\right)}^{r_{1}} \sum_{k=j}^{\infty} p^{(j-k)\left(n / q_{1}^{\prime}-n / s-\beta\right) r_{1}} \\
& \leq C\|f\|_{\dot{K}_{q_{2}}^{\beta, r_{1}}}^{r_{1}}\left(\mathbb{Q}_{p}^{n}\right)^{n}
\end{aligned}
$$

Case 2: When $r_{1}>1$, applying Hölder's inequality, we have

$$
\begin{aligned}
\left\|H_{\Omega, \alpha}^{p, b} f\right\|_{\dot{K}_{q_{2}}^{\beta, r_{2}}\left(\mathbb{Q}_{p}^{n}\right)}^{r_{1}} \leq & C \sum_{k=-\infty}^{\infty}\left(\sum_{j=-\infty}^{k} p^{j \beta} p^{(j-k)\left(n / q_{1}^{\prime}-n / s-\beta\right)}\left\|f_{j}\right\|_{L^{q_{1}}\left(\mathbb{Q}_{p}^{n}\right)}^{r_{1}}\right)^{r_{1}} \\
\leq & C \sum_{k=-\infty}^{\infty} \sum_{j=-\infty}^{k} p^{j \beta r_{1}}\left\|f_{j}\right\|_{L^{q_{1}}\left(\mathbb{Q}_{p}^{n}\right)}^{r_{1}} p^{(j-k)\left(n / q_{1}^{\prime}-n / s-\beta\right) r_{1} / 2} \\
& \times\left(\sum_{j=-\infty}^{k} p^{(j-k)\left(n / q_{1}^{\prime}-n / s-\beta\right) r_{1}^{\prime} / 2}\right)^{r_{1} / r_{1}^{\prime}} \\
\leq & C \sum_{j=-\infty}^{\infty} p^{j \beta r_{1}}\left\|f_{j}\right\|_{L^{q_{1}}}^{r_{1}}\left(\mathbb{Q}_{p}^{n}\right) \sum_{k=j}^{\infty} p^{(j-k)\left(n / q_{1}^{\prime}-n / s-\beta\right) r_{1} / 2} \\
\leq & C\|f\|_{\dot{K}_{q_{1}}^{\beta, r_{1}}\left(\mathbb{Q}_{p}^{n}\right)}^{r_{1}} .
\end{aligned}
$$

Theorem 3.3 Let $1 \leq q_{1}, q_{2}<\infty, 0<r_{1} \leq r_{2}<\infty$. Also, let $\frac{1}{q_{1}}-\frac{1}{q_{2}}=\frac{\delta+\alpha}{n}, s>q_{1}^{\prime}, \frac{1}{q_{1}^{\prime}}-\frac{1}{t}=\frac{1}{s}$, $\lambda \geq 0$ and $0<\delta<1$. If $n\left(\frac{1}{q_{1}^{\prime}}-\frac{1}{s}\right)+\lambda>\beta$, then the inequality

$$
\left\|H_{\Omega, \alpha}^{p, b} f\right\|_{M \dot{K}_{r_{2}, q_{2}}^{\beta, \lambda}\left(\mathbb{Q}_{p}^{n}\right)} \leq C\|f\|_{M \dot{K}_{r_{1}, q_{1}}^{\beta, \lambda}\left(\mathbb{Q}_{p}^{n}\right)},
$$

holds for all $\Omega \in L^{s}\left(S_{\mathbf{0}}(\mathbf{0})\right), b \in \Lambda_{\delta}\left(\mathbb{Q}_{p}^{n}\right)$, and $f \in L_{\mathrm{loc}}^{q_{1}}\left(\mathbb{Q}_{p}^{n}\right)$.

Proof of Theorem 3.3 The proof follows from standard analysis performed in our previous theorems. So, we omit the details.

\section{Acknowledgements}

Researchers supporting Project number (RSP-2020/33), King Saud University, Riyadh, Saudi Arabia.

\section{Funding}

No specific funding received for this work.

\section{Availability of data and materials}

Data sharing not applicable to this article as no data-sets were generated or analysed during the current study.

\section{Competing interests}

The authors declare that they have no competing interests.

\section{Authors' contributions}

Formal analysis, NS, AH; investigation, NS, AH; resources, IK, AA, NNH; funding acquisition, AA, NNH; supervision, AH, IK. All authors read and approved the final manuscript. 


\section{Author details}

'Department of Mathematics, Quaid-I-Azam University, 45320, Islamabad, 44000, Pakistan. ${ }^{2}$ Department of Mathematics, University of Kotli Azad Jammu and Kashmir, Kotly, Pakistan. ${ }^{3}$ Department of Mathematics, College of Science Al-Zulfi, Majmaah University, Al-Majmaah 11952, Saudi Arabia. ${ }^{4}$ Department of Basic Sciences, College of Science and Theoretical Studies, Saudi Electronic University, Riyadh 11673, Saudi Arabia.

\section{Publisher's Note}

Springer Nature remains neutral with regard to jurisdictional claims in published maps and institutional affiliations.

\section{Received: 12 December 2020 Accepted: 10 June 2021 Published online: 21 July 2021}

\section{References}

1. Albeverio, S., Karwowshi, W.: A random walk on p-adics-the generator and its spectrum. Stoch. Process. Appl. 53(1), $1-22(1994)$

2. Avestisov, A.V., Bikulov, A.H., Kozyrev, S.V., Osipov, V.A.: p-Adic models of ultrametric diffusion constrained by hierarchical energy landscape. J. Phys. A, Math. Gen. 35, 177-189 (2002)

3. Avestisov, A.V., Bikulov, A.H., Osipov, V.A.: p-Adic description of characteristic relaxation in complex systems. J. Phys. A, Math. Gen. 36, 4239-4246 (2003)

4. Christ, M., Grafakos, L.: Best constants for two non convolution inequalities. Proc. Am. Math. Soc. 123, 1687-1693 (1995)

5. Chuong, N.M., Duong, D.V.: Weighted Hardy-Littlewood operators and commutators on $p$-adic function spaces P-Adic Numb. Ultrametr. Anal. Appl. 5, 65-82 (2013)

6. Dubischar, D., Gundlach, V.M., Steinkamp, O., Khrennikov, A.: A p-adic model for the process of thinking disturbed by physiological and information noise. J. Theor. Biol. 197(4), 451-467 (1999)

7. Faris, W.G.: Weak Lebesgue spaces and quantum mechanical binding. Duke Math. J. 43, 365-373 (1976)

8. Fu, Z.W., Lu, S.Z., Zhao, F.Y.: Commutators of $n$-dimensional rough Hardy operator. Sci. China Math. 54(1), 95-104 (2011)

9. Fu, Z.W., Wu, Q.Y., Lu, S.Z.: Sharp estimates of p-adic Hardy and Hardy-Littlewood-Pólya operators. Acta Math. Sin. 29, 137-150 (2013)

10. Gao, G., Zhong, Y.: Some estimates of Hardy operators and their commutators on Morrey-Herz spaces. J. Math. Inequal. 11(1), 49-58 (2017)

11. Gürbüz, F.: Sublinear operators with rough kernel generated by Calderón-Zygmund operators and their commutators on generalized Morrey spaces. Math. Notes 101(3), 429-442 (2017)

12. Gürbüz, F.: Parabolic local Campanato estimates for commutators of parabolic fractional maximal and integral operators with rough kernel. Filomat 34(4), 1147-1156 (2020)

13. Hardy, G.H.: Note on a theorem of Hilbert. Math. Z. 6, 314-317 (1920)

14. Herz, C.S.: Lipschitz spaces and Bernstein's theorem on absolutely convergent Fourier transforms. J. Math. Mech. 18, 283-323 (1968/69)

15. Ho, K.-P.: Extrapolation to Herz spaces with variable exponents and applications. Rev. Mat. Complut. 33, 437-463 (2020)

16. Hussain, A., Ahmed, M.: Weak and strong type estimates for the commutators of Hausdorff operator. Math. Inequal. Appl. 20(1), 49-56 (2017)

17. Hussain, A., Gao, G.: Multidimensional Hausdorff operators and commutators on Herz-type spaces. J. Inequal. Appl. 2013, 594 (2013)

18. Hussain, A., Sarfraz, N.: The Hausdorff operator on weighted p-adic Morrey and Herz type spaces. P-Adic Numb. Ultrametr. Anal. Appl. 11(2), 151-162 (2019)

19. Hussain, A., Sarfraz, N.: Optimal weak type estimates for $p$-adic Hardy operator. P-Adic Numb. Ultrametr. Anal. Appl. 12(1), 12-21 (2020)

20. Hussain, A., Sarfraz, N.: Boundedness of weighted multilinear p-adic Hardy operator on Herz type spaces. arXiv:2003.02009 [math.CA]

21. Hussain, A., Sarfraz, N., Gürbüz, F.: Sharp weak bounds for $p$-adic Hardy operators on $p$-adic linear spaces. arXiv:2002.08045 [math.CA]

22. Khrennikov, A.: p-Adic Valued Distributions in Mathematical Physics and Its Applications, vol. 309. Kluwer Academic, Dordrecht (1994)

23. Kochubei, A.N.: Stochastic integrals and stochastic differential equations over the field of $p$-adic numbers. Potential Anal. 6, 105-125 (1997)

24. Kozyrev, S.V.: Methods and applications of ultrametric and $p$-adic analysis: from wavelet theory to biophysics. Proc. Steklov Inst. Math. 274, 1-84 (2011)

25. Liu, R.H., Zhou, J.: Sharp estimates for the $p$-adic Hardy type operator on higher-dimensional product spaces. J. Inequal. Appl. 2017, 219 (2017)

26. Lu, S., Xu, L.: Boundedness of rough singular integral operators on the homogeneous Morrey-Herz spaces. Hokkaido Math. J. 34, 299-314 (2005)

27. Lu, S., Yang, D., Hu, G.: Herz Type Spaces and Their Applications. Science Press, Beijing (2008)

28. Lu, S.Z., Yang, D.C., Zhao, F.Y.: Sharp bounds for Hardy type operators on higher dimensional product spaces. J. Inequal. Appl. 2013, 148 (2013)

29. Persson, L.-E., Samko, S.G.: A note on the best constants in some Hardy inequalities. J. Math. Inequal. 9(2), 437-447 (2015)

30. Sarfraz, N., Gürbüz, F.: Weak and strong boundedness for $p$-adic fractional Hausdorff operator and its commutators (2019). arXiv:1911.09392v1

31. Sarfraz, N., Hussain, A.: Estimates for the commutators of $p$-adic Hausdorff operator on Herz-Morrey spaces. Mathematics 7(2), 127 (2019) 
32. Varadarajan, V.S.: Path integrals for a class of $p$-adic Schrodinger equations. Lett. Math. Phys. 39(2), 97-106 (1997)

33. Vladimirov, V.S.: Tables of integrals of complex valued functions of $p$-adic arguments. Proc. Steklov Inst. Math. 284 $1-59$ (2014)

34. Vladimirov, V.S., Volovich, I.V., Zelenov, E.I.: p-Adic Analysis and Mathematical Physics. World Scientific, Singapore (1994)

35. Volosivets, S.S.: Weak and strong estimates for rough Hausdorff type operator defined on $p$-adic linear space. P-Adic Numb. Ultrametr. Anal. Appl. 9(3), 236-241 (2017)

36. Wu, Q.Y.: Boundedness for commutators of fractional p-adic Hardy operator. J. Inequal. Appl. 2012, 293 (2012)

37. Wu, Q.Y., Mi, L., Fu, Z.W.: Boundedness of $p$-adic Hardy operators and their commutators on $p$-adic central Morrey and BMO spaces. J. Funct. Spaces Appl. 2013, Article ID 359193 (2013)

38. Xiao, J.: $L^{p}$ and BMO bounds of weighted Hardy-Littlewood averages. J. Math. Anal. Appl. 262, 660-666 (2001)

39. Yee, T.L., Ho, K.-P.: Hardy's inequalities and integral operators on Herz-Morrey spaces. Open Math. 18, 106-121 (2020)

\section{Submit your manuscript to a SpringerOpen ${ }^{\circ}$ journal and benefit from:}

- Convenient online submission

Rigorous peer review

Open access: articles freely available online

High visibility within the field

- Retaining the copyright to your article

Submit your next manuscript at $\gg$ springeropen.com 